\title{
THE EARLY TECHNICAL DEVELOPMENT OF THE VLA
}

\author{
GEORGE W. SWENSON, JR. \\ University of Mlinois, 1406 W. Green Street, Urbana, IL 61801
}

In the spring of 1964, having qualified for a sabbattical leave from the University of Illinois and having recently completed two years as Chairman of the Visiting Committee of the National Radio Astronomy Observatory, I inquired of the NRAO staff as to whether a desk and possibly some augmentation of my University half-salary might be available at Green Bank for the following academic year. Instead I was invited to join the staff as a fulltime employee and to take a coordinating role in the development of the "very large array" as Chairman of the Design Committee. The University granted me a year's leave of absence which eventually stretched to four years.

The nucleus of the development team already existed at Green Bank, including Hein Hvatum, Warren Tyler, David Hogg, Campbell Wade, and of course, David Heeschen, the Director of the Observatory, who set the parameters and goals of the study. This group was soon joined by Barry Clark and Sander Weinreb, both of whom made sustained, outstanding contributions which were major elements in the success of the program. During the development stage, a number of scientists and engineers joined the team for varying periods, including Naresh Mathur, Torleiv Orhaug, Leonard Chow, Nigel Keen and Marc Vinocur. Advice and assistance was regularly supplied by outside experts such as Marshall Cohen, William Erickson, Gart Westerhout, Bernard Burke, Alan Moffet, James Douglas, J. L. Yen, Robert MacPhie, E. J. Blum, Jan Hogbom and Frank Drake.

The program was motivated by the universally-perceived need for higher angular resolution in radio astronomical observations. Many efforts were under way to make major improvements in available resolution, which at the beginning of this epoch was of the order of tens of arc-minutes, but there was no consensus about the best way to achieve it. The Mills cross and its derivatives, consisting of two long, narrow filled arrays, usually orthogonal, whose outputs are multiplied to produce a pencil beam, seemed to have the most adherents. Very large instruments of this nature were in the planning stage in Europe (the Benelux Cross) and under construction in Australia (Molonglo Cross), both to produce resolution of the order of one arcminute. The other technique which was viewed as promising was the array of interferometers using earth rotation to increase the range of effective baseline vectors available from a single physical baseline. This general principle had been known and used since the very early days of radio astronomy, primarily in England and Australia, but mostly in the context of discrete source surveying and astrometry. At Caltech an interferometer existed on orthogonal railroad tracks which was in principle capable of full source synthesis but up to this time had been engaged in necessary preliminaries such as catalogueing and in astrometry in support of identification of radio sources with optical objects. At this time, then, despite the outstanding work of many interferometrists, 
particularly at Cambridge, Jodrell Bank, the Royal Radar Establishment and Caltech, there had been no really convincing demonstration of earth rotation synthesis as a technique for high resolution mapping.

In 1962 Ryle and Neville published a map of the north polar region with a single physical interferometer, using earth rotation. In 1963 Bernard Rowson published a particularly lucid explanation of the technique. At this time both radio astronomers and antenna engineers were, for the most part, accustomed to thinking in terms of antenna beamwidths, sidelobe levels, brightness distributions, collecting areas and gains. Spatial frequencies, visibility functions and spectral sensitivities were unknown or only vaguelyunderstood concepts. By 1964 there was growing realization that earth rotation synthesis was to assume greater importance. Everyone had studied Fourier series in college, electrical engineers used Laplace transforms routinely in causal situations, opticians recognized the relation of the Fraunhofer diffraction problem to the Fourier integral, but the Fourier transform was not an everyday tool for radio astronomers. Seminal papers by Ron Bracewell and an excellent little book by Roger Jennison helped in the philosophical conversion from the angular domain to the $\mathrm{U}-\mathrm{V}$ plane.

When the VLA Design Group was activated at Green Bank a large interferometer was already under construction, consisting of the existing 85-foot equatorial antenna in addition to a new one, with several observing stations for the latter along a northeast-southwest baseline long enough to produce 8 -arcsecond fringes at eleven centimeters wavelength. This instrument was brought into use during 1965 and immediately demonstrated that stable fringes could be obtained at that resolution, even in the rather unsuitable atmosphere and topography of the Allegheny Mountains. The existence of this far-sighted project probably indicates that interferometric synthesis was viewed as the front-running technique for the VLA even in 1962, when the budgeting for the interferometer must have been done. Even if the filled array had won out, the understanding of the atmosphere gained from the interferometer would have been very valuable.

The design goals for the VlA initially specified by the Director were a resolution of one arcsecond for a source on the celestial equator, with a maximum sidelobe level $30 \mathrm{~dB}$ below the main beam. To determine if this was feasible required some experimentation, both in the field and on the computer. Several technical questions arose: Would the atmosphere sustain this resolution? How would phase-stable local oscillator signals be transmitted over the necessary long distances, and would the atmosphere permit use of radio links for this purpose? How would the necessary time delays for the i-f signals be provided and how would the wide band i-f communication channels be implemented? What should be the geometrical configuration of the array? No specification was set for the overall sensitivity of the array; it was realized that for any economically-feasible antenna size the instrument would be sensitivity limited, so the antennas should be as large as possible.

To address further the atmospheric questions a longer interferometer baseline was needed. A site was available (Spencer's Ridge) 12 kilometers from the Green Bank antennas, with line-of-sight intervisibility. Following a technique developed by Moody Thompson of the Department of Commerce Boulder Laboratories a round-trip, phase-locked radio link was established 
to synchronize the local oscillators at the two sites and an inexpensive 42foot equatorial paraboloid was acquired. This dish had acceptable efficiency at $11 \mathrm{~cm}$ wavelength, had four hours of hour-angle coverage, and manuallyadjusted declination. This setup immediately demonstrated the feasibility of one arcsecond resolution and radio-link phase synchronization, at least at that site. A series of observations was made to gather statistics on phase stability, concurrently with water vapor and other atmospheric properties in the vicinity. It was not long before the astronomers became aware of the success of the radio-link experiment, whereupon the equipment was effectively removed from engineering control and diverted to scientific observing. This was satisfactory, as it produced the desired data in any case.

The $12 \mathrm{~km}$ interferometer required long time delays in the i-f channels. As in the shorter Green Bank interferometer the delays were provided by bulky binary arrays of coaxial cable sections, literally tons of it, housed in the control building along with the necessary amplifiers and equalizers. The thought of extrapolating this to dozens or scores of baselines was intimidating, so the ony apparently-feasible alternative, ultrasonic quartz delay devices, was investigated with the assistance of industrial contractors. This would have been a complicated and expensive and probably troublesome solution, but at the time the VLA proposal was issued (Volumes 1 and 2) in January 1967 the ultrasonic quartz technology was indicated as the method of choice. This was a nagging concern through the early years of the program, but fortunately the arrival on the scene of suitable digital correlator and shiftregister technology made digital delay lines the obvious choice well before actual construction started. The delay problem was, of course, intimately related to the $\mathrm{i}-\mathrm{f}$ bandwidth question, which was similarly a matter of concern at the time of the Proposal.

In December, 1985 VLA Report No. 1 was issued, just about 18 months after the Design Group was formed. It presented arguments for the alternative types of array that might satisfy the adopted specifications, and concluded that the earth rotation synthesis array was the only realistic choice. Thereafter all effort was directed toward that option.

Having made that decision, the dominant remaining question concerned the array configuration and the number of antennas. One had to assume some budgetary limit on cost and then to compromise the size and number of antennas, bearing in mind the requirements on resolution and sidelobe levels. The cost of antennas varies as some power of the size and directly (more or less) as their number. The cost of correlators varies as the square and the cost of delay lines directly as the number of antennas, and so on. Computer programs were written to plot in the U-V plane the diurnal tracks of the many antenna pairs in an array, to determine the corresponding "beam pattern" on the celestial sphere by Fourier transformation, and then to compute the resulting sidelobe-to-main-beam levels. We tried many configurations: crosses, tees, circles, ellipses, random patterns, Y's. Eventually the Y won out, not only by virtue of its excellent transfer function in the U-V plane, but also because it accommodated a convenient system for changing the geometrical parameters of the array and for moving antennas to storage or maintenance facilities. After the $\mathrm{Y}$ was chosen its parameters were subjected to much further tuning, by varying its orientation slightly, by changing antenna positions along the arms, and by changing numbers of antennas. 
Concurrently, studies of antenna structural and electromagnetic design were carried out, as well as preliminary studies of low noise amplifiers, i-f transmission facilities, antenna mobility systems, and a host of other details. It was decided that the largest feasible antenna from the aspects of cost and performance was an 85-foot paraboloid on an alt-azimuth mount. Numerous successful prototypes existed and a suitable design was well within the state of the art.

Site searches began early in the program, first by setting geographical constraints: high, dry Southwestern U.S.A., then by study of topographic maps for suitable terrain and isolation from noise sources, and then by personal inspection of the most promising places. Atmospheric parameters were measured at Marfa, Texas; Tucson, Arizona and Magdalena, New Mexico. The results confirmed that the higher sites had lower total water vapor content, in agreement with earlier studies by others. Meanwhile, experience with the Green Bank interferometer showed better phase stability with lower humidity. Fortunately, the highest site on the short list, Magdalena, also had excellent characteristics in other respects. Inspections by various NRAO staff all resulted in the opinion that this was the best place for the VLA.

In January, 1967 NRAO issued the VLA Proposal, Volumes I and II. It described a Y-shaped array of 36 25-meter paraboloids on railway-mobile mounts, each arm of the $Y 21 \mathrm{Km}$ long. Maximum resolution was 1", with $30 \mathrm{~dB}$ sidelobes, at $11 \mathrm{~cm}$ wavelength. Three more compact arrays are also included. The total cost was to be $\$ 51.9$ million. Not very much was said about spectral line capability, though a brief analysis was made of systems of restricted capability. Today this proposal looks rather primitive, but in its day it had to be considered bold and imaginative. In February, 1967 NRAO announced the proposal to the public and in April it was described in Sky and Telescope. As described in the previous paper there remained many years of struggle before Governmental authority to proceed was granted. The intervening years were well spent by the technical and scientific staffs of the program. Digitization of the entire system solved several of the problems described above. Advances in low-noise amplifier technology and in other electronic areas have made possible order of magnitude improvements in operating frequency and hence in angular resolution, as well as in sensitivity. Probably the most unexpected development, which can only be described as a breakthrough, is the appearance of the sophisticated post-observational data processing algorithms which have permitted improvements in the dynamic range of the synthesized images by two orders of magnitude. The instrument has proven to be flexible and robust, adaptable to assignments never contemplated by those who conceived it.

Even though there had as yet appeared no elegant solutions to such vexing problems as the i-f delay system, the broadband i-f transmission system and spectroscopic methodology, and even though much engineering study and design of anternnas, electronics, computers and mobility system remained to be done, after the first two volumes of the Proposal had been issued there remained no doubt in the minds of the development team that the VLA could be built to meet the specifications in effect at the time.

Having left the program in 1968, I take avuncular delight in the accomplishments of those who have, since then, made this instrument the 
outstanding success it is. It is among the very few "big science" instruments which have greatly exceeded its designers' expectations.

J. P. Hamaker: There is another breakthrough that must have occurred well after you had begun to get serious about the project; and that is the discovery of the Fast-Fourier-Transform algorithm. I wonder what your views on the computing problem were prior to its existence.

George Swenson: Cooley and Tukey published their famous paper in April, 1965 , and it was quoted in the second volume of the VLA proposal which was issued in January 1967. For the instrument envisioned at that time. The map reconstruction ("Fourier inversion") computing load was expected to be substantially less than the loads for data reduction and data sorting. Thus, little was said about the former in the proposal. Of course, that was before the enormous benefits of subsequently developed image-processing algorithms were appreciated. 